\title{
Supporting Information \\ Measurement of Net Rate Constants from Enzyme \\ Progress Curves without Curve Fitting
}

\author{
Mark W. Ruszczycky ${ }^{1}$ and Hung-wen Liu ${ }^{1,2}$ \\ ${ }^{1}$ Division of Chemical Biology and Medicinal Chemistry, College of Pharmacy, \\ University of Texas at Austin \\ ${ }^{2}$ Department of Chemistry, University of Texas at Austin
}

\section{Contents}

$\begin{array}{ll}\text { S1 Notes on the equilibrium state } & \text { S1 }\end{array}$

$\begin{array}{ll}\text { S2 Inductive proof for } n>2 & \text { S2 }\end{array}$

S3 Special cases: $n \leq 2 \quad$ S2

S4 Expressing $k_{\text {cat }}$ in terms of $\bar{e}_{1} \quad$ S3

S5 Simulation details for the mock experiments $\quad$ S5

\section{S1 Notes on the equilibrium state}

The time-derivatives of all species concentrations in the complete enzymatic reaction are equal to 0 at equilibrium. Restricted to the species in the subsequence (2), this implies $\dot{x}=0$ and $\dot{e}_{i}=0$ for all $i$ where $1 \leq i \leq n$. Summing over Eqs. (4) and applying the equilibrium condition yields $e_{n}^{*}=$ 0 , where $e_{i}^{*}$ denotes an equilibrium concentration of $E_{i}$. Combining this result with Eq. (4d) implies $e_{n-1}^{*}=0$. With these results in hand, one may proceed inductively to show that $e_{i}^{*}=0$ whenever $1<i \leq n$. Similarly, $e_{1}^{*} x^{*}=0$ in the equilibrated state. However, while at least one of $e_{1}^{*}$ and $x^{*}$ is 0 , nothing more can be said without further specification of the model. 


\section{S2 Inductive proof for $n>2$}

It was already shown in the main text that (3) holds for $E_{n}$ and $E_{n-1}$ when $n>2$ in the subsequence (2). This proves the base case, and if $n=3$, then there is nothing left to do. Therefore, assume the inductive hypothesis that (3) holds for $E_{i}$ and $E_{i+1}$ for some $i$ where $2<i<n$. Since $2<i<n$, Eq. (4c) applies. Integration of (4c) and taking the limit as $t \rightarrow \infty$ provides

$$
0=k_{i-1, i} \lim _{t \rightarrow \infty} \int_{0}^{t} e_{i-1}(\tau) d \tau-\left(k_{i, i-1}+k_{i, i+1}\right) A_{i}+k_{i+1, i} A_{i+1} .
$$

Therefore, the improper integral in (14) exists for $i-1$, since $A_{i}$ and $A_{i+1}$ exist by the inductive hypothesis. Furthermore, $A_{i}=\Delta x_{\infty} / k_{i}^{\prime}$ and $A_{i+1}=$ $\Delta x_{\infty} / k_{i+1}^{\prime}$ by (3), and it follows that

$$
\begin{aligned}
0 & =k_{i-1, i} A_{i-1}-\Delta x_{\infty} \frac{k_{i, i-1}}{k_{i}^{\prime}}-\Delta x_{\infty} \frac{k_{i, i+1}}{k_{i}^{\prime}}+\Delta x_{\infty} \frac{k_{i+1, i}}{k_{i+1}^{\prime}}, \\
& =\frac{k_{i-1, i} A_{i-1}}{\Delta x_{\infty}}-\frac{k_{i, i-1}}{k_{i}^{\prime}}-k_{i, i+1}\left(\frac{k_{i+1, i}+k_{i+1}^{\prime}}{k_{i, i+1} k_{i+1}^{\prime}}\right)+\frac{k_{i+1, i}}{k_{i+1}^{\prime}}, \\
& =\frac{A_{i-1}}{\Delta x_{\infty}}-\frac{k_{i, i-1}}{k_{i-1, i} k_{i}^{\prime}}-\frac{1}{k_{i-1, i}}=\frac{A_{i-1}}{\Delta x_{\infty}}-\frac{1}{k_{i-1}^{\prime}},
\end{aligned}
$$

since by Cleland's recursive expression $k_{i-1}^{\prime}=k_{i-1, i} k_{i}^{\prime} /\left(k_{i, i-1}+k_{i}^{\prime}\right) .{ }^{1}$ Hence, $A_{i-1}$ is positive and $k_{i-1}^{\prime}=\Delta x_{\infty} / A_{i-1}$. By hypothesis, $k_{(i-1)+1}^{\prime}=\Delta x_{\infty} / A_{i}$. Consequently, (3) holds by induction for all $i>1$ when $n>2$.

\section{S3 Special cases: $n \leq 2$}

\section{Case where $n=1$}

If $n=1$, then the subsequence in (2) reduces to

$$
\cdots E_{1}+X \stackrel{k_{12}}{\longrightarrow} E_{2} \cdots
$$

and only the single differential equation, $\dot{x}=-k_{12} x e_{1}$, suffices for the theory. Integration and taking the limit as $t \rightarrow \infty$ thus yields $\Delta x_{\infty}=k_{12} B_{1}$ whenever $X$ satisfies (9). Hence, $B_{1}$ (see (19)) exists and is positive since $\Delta x_{\infty}, k_{12}>0$. Consequently, $k_{12}=\Delta x_{\infty} / B_{1}$, which is a special case of (20). 


\section{Case where $n=2$}

If $n=2$, then the subsequence in (2) reduces to

$$
\cdots E_{1}+X \underset{k_{21}}{\stackrel{k_{12}}{\rightleftharpoons}} E_{2} \stackrel{k_{23}}{\longrightarrow} E_{3} \cdots
$$

such that if $E_{3}=E_{1}$, then this just becomes the Briggs-Haldane model of steady-state kinetics. ${ }^{2,3}$ The associated system of two differential equations is given by

$$
\begin{aligned}
\dot{x} & =-k_{12} x e_{1}+k_{21} e_{2}, \\
\dot{e}_{2} & =k_{12} x e_{1}-\left(k_{21}+k_{23}\right) e_{2},
\end{aligned}
$$

and thus $\dot{e}_{2}+\dot{x}=-k_{23} e_{2}$. Integrating and applying the hypotheses in (9), one finds $\Delta x_{\infty}=k_{23} A_{2}$ such that the positive improper integral $A_{2}$ exists. Since $E_{2} \rightarrow E_{3}$ is modeled as irreversible, $k_{2}^{\prime}=k_{23}$ so that $k_{2}^{\prime}=\Delta x_{\infty} / A_{2}$. It then follows that

$$
\left(\frac{k_{c a t}}{K_{M}}\right)_{X}=\phi_{1} \frac{k_{12} k_{23}}{k_{21}+k_{23}}=\phi_{1} \frac{\Delta x_{\infty}}{B_{1}},
$$

where positive $B_{1}$ (see (19)) exists and $\phi_{1}$ is defined as in the main text. Furthermore, if $E_{3}=E_{1}$, then $\phi_{1}=1$ and $\left(k_{\text {cat }} / K_{M}\right)_{X}$ is the $k_{\text {cat }} / K_{M}$ of the Briggs-Haldane model.

\section{S4 Expressing $k_{\text {cat }}$ in terms of $\bar{e}_{1}$}

According to the definitions (23) and (24), it follows that

$$
\frac{\bar{v}(t)}{e_{0}-\bar{e}_{1}(t)}=\frac{x(0)-x(t)}{e_{0} t-\int_{0}^{t} e_{1}(\tau) d \tau}, \quad t>0,
$$

where $e_{0}$ is the constant total enzyme concentration. Integration is a linear operation; hence, given the model in (21), the denominator of the righthand side of (S4) is given by

$$
e_{0} t-\int_{0}^{t} e_{1}(\tau) d \tau=\int_{0}^{t}\left(e_{0}-e_{1}(\tau)\right) d \tau=\int_{0}^{t} \sum_{i=2}^{n} e_{i}(\tau) d \tau=\sum_{i=2}^{n} \int_{0}^{t} e_{i}(\tau) d \tau \text {. }
$$

Thus the positive limit of this summation as $t \rightarrow \infty$ exists whenever the limits $A_{i}$ all exist for $1<i \leq n$. Consequently,

$$
\lim _{t \rightarrow \infty} \frac{\bar{v}(t)}{e_{0}-\bar{e}_{1}(t)}=\frac{\lim _{t \rightarrow \infty}(x(0)-x(t))}{\lim _{t \rightarrow \infty} \sum_{i=2}^{n} \int_{0}^{t} e_{i}(\tau) d \tau}=\frac{x_{0}}{\sum_{i=2}^{n} A_{i}}=k_{c a t} .
$$


Table S1: Summary of the kinetic parameters and starting conditions for the simulations both with and without a competitive inhibitor. The total initial concentrations of $X$ (i.e., $\mathrm{O}_{2}$ ), $S$ and enzyme were $0.5,0.75$ and $0.5 \mathrm{mM}$, respectively; however, before $\mathrm{O}_{2}$ is rapidly combined with the enzyme/substrate mixture, $E_{1}$, $E_{6}$ and $S$ form an equilibrated mixture having the listed concentrations. In the presence of the competitive inhibitor $W$, which was mocked as a contaminant in the solution containing $X$, the initial concentrations of $W$ and dead-end complex $E_{7}$ were 0.5 and $0.0 \mathrm{mM}$, respectively.

\begin{tabular}{ccccccc}
\hline \multicolumn{3}{c}{ Concentrations } & & \multicolumn{3}{c}{ Rate Constants } \\
Param. & Value & Units & & Param. & Value & Units \\
\cline { 1 - 4 }$s(0)$ & 0.5685 & $\mathrm{mM}$ & & $k_{65}$ & 10.0 & $\mathrm{mM}^{-1} \mathrm{~s}^{-1}$ \\
$x(0)$ & 0.5 & $\mathrm{mM}$ & & $k_{61}$ & 10.0 & $\mathrm{mM}^{-1} \mathrm{~s}^{-1}$ \\
$p(0)$ & 0.0 & $\mathrm{mM}$ & & $k_{16}$ & 10.0 & $\mathrm{~s}^{-1}$ \\
$e_{1}(0)$ & 0.1815 & $\mathrm{mM}$ & & $k_{12}$ & 50.0 & $\mathrm{mM}^{-1} \mathrm{~s}^{-1}$ \\
$e_{2}(0)$ & 0.0 & $\mathrm{mM}$ & & $k_{21}$ & 10.0 & $\mathrm{~s}^{-1}$ \\
$e_{3}(0)$ & 0.0 & $\mathrm{mM}$ & & $k_{23}$ & 50.0 & $\mathrm{~s}^{-1}$ \\
$e_{4}(0)$ & 0.0 & $\mathrm{mM}$ & & $k_{32}$ & 10.0 & $\mathrm{~s}^{-1}$ \\
$e_{5}(0)$ & 0.0 & $\mathrm{mM}$ & & $k_{34}$ & 30.0 & $\mathrm{~s}^{-1}$ \\
$e_{6}(0)$ & 0.3185 & $\mathrm{mM}$ & & $k_{43}$ & 0.0 & $\mathrm{~s}^{-1}$ \\
& & & & $k_{45}$ & 10.0 & $\mathrm{~s}^{-1}$ \\
& & & & $k_{54}$ & 0.0 & $\mathrm{~s}^{-1}$ \\
& & & & $k_{56}$ & 10.0 & $\mathrm{~s}^{-1}$ \\
\cline { 6 - 8 }$w(0)$ & 0.5 & $\mathrm{mM}$ & & $k_{17}$ & 50.0 & $\mathrm{mM}^{-1} \mathrm{~s}^{-1}$ \\
$e_{7}(0)$ & 0.0 & $\mathrm{mM}$ & & $k_{71}$ & 10.0 & $\mathrm{~s}^{-1}$ \\
\hline
\end{tabular}




\section{S5 Simulation details for the mock experiments}

The nine progress curves for the uninhibited mock experiment were obtained by numerically solving the following system of nine coupled, nonlinear, ordinary differential equations,

$$
\begin{aligned}
\dot{s} & =k_{16} e_{1}-k_{61} s e_{6}, \\
\dot{x} & =k_{21} e_{2}-k_{12} x e_{1}, \\
\dot{p} & =k_{56} e_{5}-k_{65} p e_{6}, \\
\dot{e}_{1} & =k_{61} s e_{6}-\left(k_{12} x+k_{16}\right) e_{1}+k_{21} e_{2}, \\
\dot{e}_{2} & =k_{12} x e_{1}-\left(k_{21}+k_{23}\right) e_{2}+k_{32} e_{3}, \\
\dot{e}_{3} & =k_{23} e_{2}-\left(k_{32}+k_{34}\right) e_{3}+k_{43} e_{4}, \\
\dot{e}_{4} & =k_{34} e_{3}-\left(k_{45}+k_{43}\right) e_{4}+k_{54} e_{5}, \\
\dot{e}_{5} & =k_{45} e_{4}-\left(k_{54}+k_{56}\right) e_{5}+k_{65} p e_{6}, \\
\dot{e}_{6} & =k_{56} e_{5}-\left(k_{65} p+k_{61} s\right) e_{6}+k_{16} e_{1} .
\end{aligned}
$$

The eleven progress curves for the inhibited mock experiment were obtained by numerically solving the following system of eleven coupled, nonlinear, ordinary differential equations,

$$
\begin{aligned}
\dot{s} & =k_{16} e_{1}-k_{61} s e_{6}, \\
\dot{x} & =k_{21} e_{2}-k_{12} x e_{1}, \\
\dot{p} & =k_{56} e_{5}-k_{65} p e_{6}, \\
\dot{w} & =k_{71} e_{7}-k_{17} w e_{1} \\
\dot{e}_{1} & =k_{61} s e_{6}-\left(k_{12} x+k_{16}+k_{17} w\right) e_{1}+k_{21} e_{2}+k_{71} e_{7}, \\
\dot{e}_{2} & =k_{12} x e_{1}-\left(k_{21}+k_{23}\right) e_{2}+k_{32} e_{3}, \\
\dot{e}_{3} & =k_{23} e_{2}-\left(k_{32}+k_{34}\right) e_{3}+k_{43} e_{4}, \\
\dot{e}_{4} & =k_{34} e_{3}-\left(k_{45}+k_{43}\right) e_{4}+k_{54} e_{5}, \\
\dot{e}_{5} & =k_{45} e_{4}-\left(k_{54}+k_{56}\right) e_{5}+k_{65} p e_{6}, \\
\dot{e}_{6} & =k_{56} e_{5}-\left(k_{65} p+k_{61} s\right) e_{6}+k_{16} e_{1}, \\
\dot{e}_{7} & =k_{17} w e_{1}-k_{71} e_{7} .
\end{aligned}
$$

All parameter values and initial conditions at $t=0$ are listed in Tbl. S1 for both mock experiments. Numerical analyses over the $t$-interval $[0,5]$ (in seconds, uninhibited) or $[0,10]$ (in seconds, inhibited) were performed using the fourth-order Runge-Kutta algorithm ${ }^{4}$ implemented in Haskell. ${ }^{5}$ Trajectories utilized step sizes of $\Delta t=0.001 \mathrm{~s}$ and were not significantly 
changed upon increasing the step size to $\Delta t=0.005 \mathrm{~s}$ indicating no signficant discretization error. The computed trajectories were integrated using trapezoids implemented in Haskell to determine the mock $\hat{A}_{i}$ and $\hat{B}_{1}$ observables. The trajectories are plotted in Figs. 2 \& 3 using Python with the Matplotlib package. ${ }^{6,7}$

\section{References}

[1] Cleland, W. W. (1975) Partition analysis and the concept of net rate constants as tools in enzyme kinetics. Biochemistry 14:3220-3224.

[2] Cook, P. F., Cleland, W. W. (2007) Enzyme Kinetics and Mechanism. Garland Science, New York.

[3] Cornish-Bowden, A. (2012) Fundamentals of Enzyme Kinetics. WileyBlackwell, Weinheim, Germany, 4th edition.

[4] Hildebrand, F. B. (1987) Introduction to Numerical Analysis. Dover, New York, 2nd edition.

[5] Hudak, P., Hughes, J., Peyton Jones, S., Wadler, P. (2007) A history of Haskell: being lazy with class. In Proceedings of the Third ACM SIGPLAN Conference on History of Programming Languages.

[6] Python Software Foundation, https://docs.python.org/3.6 (2018) Python Language Reference, version 3.6.8.

[7] Hunter, J. D. (2007) Matplotlib: a 2D graphics environment. Computing in Science \& Engineering 9:90-95. 